\title{
Investigation of Spray Patterns of Selected Sprinklers with the FMRC Drop Size Measuring System
}

\author{
HONG-ZENG YOU \\ Factory Mutual Research Corporation \\ 1151 Boston-Providence Turnpike \\ Norwood, Massachusetts 02062, USA
}

\section{ABSTRACT}

Characteristics of single sprinkler sprays for three selected upright sprinklers were investigated with the FMRC drop size measuring system. The sprays of sprinklers with nominal orifice sizes of $16.3,13.5$, and $12.7 \mathrm{~mm}$ were mapped out at water pressures of 206 and $393 \mathrm{kPa}$ and at 3.05 and $6.10 \mathrm{~m}$ below the sprinklers in a measuring sector whose bisector was perpendicular to the sprinkler pipe.

Correlations of gross drop size distributions were derived based on the measurements. The gross drop size distributions at the $3.05 \mathrm{~m}$ level could be reproduced with the data measured at the $6.10 \mathrm{~m}$ level. The gross drop size distributions of the tested sprinklers can be represented by a composite of a log-norma1 distribution and a Rosin-Rammler distribution. An economical test protocol was also developed to quantify the spray characteristics of commercial sprinklers.

\section{INTRODUCTION}

Until recently, investigation of spray characteristics remained hindered by the lack of an efficient technique for counting and sizing drops within sprinkler sprays. Two methods have been employed in the past decade to measure drop sizes of sprinkler sprays: 1) freezing the falling liquid drops with liquid nitrogen; and 2) drop analysis by stil1-photography. Both freezing and photographic methods are time consuming in counting and sizing drops. Furthermore, the photographic method may bias the results due to incorrect focusing, while the freezing method is likely to produce erroneous results caused by breaking large particles and coalescing small ones.

In 1979 FMRC acquired a computerized high-speed drop sizing and counting system. This instrument is a laser-illuminated optical array imaging device originally designed to be carried by aircraft to measure the particle size spectra of clouds and precipitation". Successive modification of the probe system from its original design, both in hardware and software, was performed to accommodate measurements under sprinkler spray ${ }^{2}$. The maximum effective sampling area of the probe is $61 \mathrm{~mm} \times 6.4 \mathrm{~mm}$, and the probe was designed to accommodate drop sizes ranging from $100 \mathrm{\mu m}$ to $6400 \mathrm{\mu m}$. The probe system was calibrated with monodisperse and polydisperse samples of solid spheres ${ }^{2,3}$. The calibration tests with monodisperse samples demonstrated that the probe 
system sized the particles in the range 100-6000 jm within 30 im and measured their velocities within $10 \%$ of the actual velocities. The calibration tests with polydisperse samples showed that the system could satisfactorily report the particle size distribution of a sample ${ }^{3}$. The detailed description of the current FMRC probe system is given in References 2, 3, and 4.

The probe system was not calibrated with water drops. However, the probe system software screened out those drops whose images were skewed from the circular image beyond a predetermined tolerance ${ }^{2}$. Therefore, the adverse effect of water drop deformation on the accuracy of the measurements reported in this study was minimized.

Detailed spray mapping is generally needed to adequately quantify a spray, because of the drastic variation in distributions within the sprinkler spray. It was found that, for tested commercial sprinklers ${ }^{2}$, a 5-min period was required to achieve good measurements for certain locations within the spray. Thus, detailed spray mapping was not economical even with the high speed drop sizing and counting system. Therefore, before conducting a large number of tests to quantify spray characteristics of commercial sprinklers, an economical test protocol capable of generating reliable information should be established.

The objectives of the present investigation were: 1) Detail mapping of the variation of spray characteristics for selected upright sprinklers at selected heights and water pressures; 2) Correlation of the spray characteristics for the selected sprinkler sprays; 3) Use of the gathered data to develop a test protocol to quantify sprinkler sprays economically and accurately.

\section{EXPERTMENTS}

In the present study, seven single sprinkler sprays were mapped out at elevations of 3.05 or $6.10 \mathrm{~m}$ below the sprinkler. The tested sprinklers were limited to three selected upright sprinklers illustrated photographically in Figure 1. A summary of the test variables is presented in Table $I$.

TABLE I. Test Variables

\begin{tabular}{ccccc} 
Case & $\begin{array}{c}\text { Water Pressure } \\
(\mathrm{kPa})\end{array}$ & $\begin{array}{c}\text { Water Discharge Rate } \\
(\text { (1iters/min) }\end{array}$ & $\begin{array}{c}\text { Nozzle Dia. } \\
(\mathrm{mm})\end{array}$ & $\begin{array}{c}\text { Vertical } \\
\text { Distance Between } \\
\text { Probe and Sprinkler } \\
\text { (m) }\end{array}$ \\
\cline { 2 - 3 } 1 & 206 & 227 & 16.3 & 3.05 \\
3 & 393 & 314 & 16.3 & 3.05 \\
4 & 206 & 227 & 16.3 & 6.10 \\
5 & 393 & 314 & 16.3 & 6.10 \\
6 & 206 & 163 & 13.5 & 3.05 \\
7 & 206 & 163 & 13.5 & 6.10 \\
& 206 & 117 & 12.7 & 3.05
\end{tabular}


The experiments were conducted in the FMRC fire test building at West Glocester, Rhode Island. Figure 2 schematically depicts the floor plan of the test setup. The test setup was located at about the floor center within a test site of $26.60 \mathrm{~m} \times 26.60 \mathrm{~m} \times 18.28 \mathrm{~m}$ high. The ceiling was $3.66 \mathrm{~m} \times 3.66 \mathrm{~m}$ square and fixed at $7.62 \mathrm{~m}$ above the floor. The tested upright sprinkler was installed on a pipe of $25.4 \mathrm{~mm}$ pipe size and centered under the ceiling; the pipe was about $0.15 \mathrm{~m}$ below the ceiling. The sprinkler was so oriented that its two supporting arms were in alignment with the pipe.

A large-scale, remote-controlled, probe-traversing apparatus was constructed to traverse the probe within the circular sector shown in Figure 2. The radius of the sector was $6.1 \mathrm{~m}$ long and the angle encompassed by the arc was 100 degrees. The traversing apparatus rested on the floor and the probe elevation could be adjusted from $1 \mathrm{~m}$ to $6.1 \mathrm{~m}$ above the floor. Different vertical distances between the probe and celling were achieved by adjusting the probe elevation.

The water flux was measured separately by automatic water $\operatorname{collectors}^{6}$ at the same locations relative to the sprinkler as with the drop size measurements. Each water collector covered a floor area of $500 \mathrm{mn} \times 500 \mathrm{~mm}$. The design and operation of the collector is given in Reference 6 .

\section{DATA ANALYSIS AND RESULTS}

In each series of the measurements, the probe was traversed azimuthally from 5 degrees to 95 degrees in increments of 10 degrees. Along each radius, measurements were conducted at six. locations about equally spaced. The outermost location in each radius was determined visually to coincide with the edge of the spray.

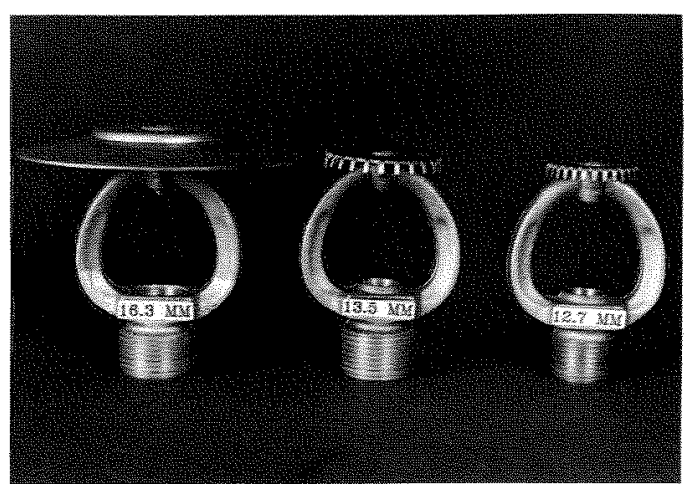

FIGURE 1. Tested upright sprinklers.

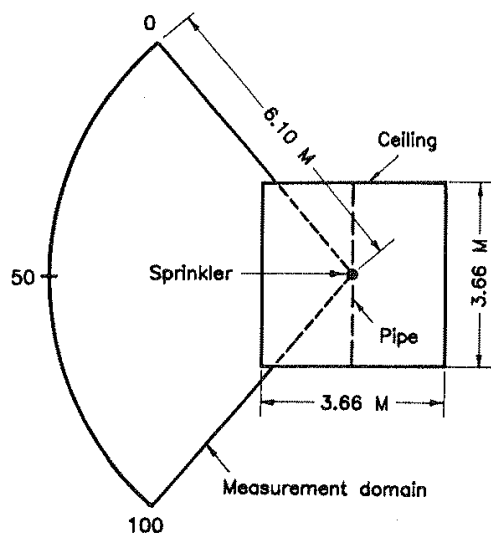

FIGURE 2. Floor plan of test setup. 


\subsection{Water Flux Distribution}

Detalled data listings of all tested cases are presented in Reference 5. Figure 3 shows the water flux distribution in the measuring sector for Case 5, to illustrate the drastic variation of water flux distributions within the sprays of real-world sprinklers.

We may divide the sector into a number of zones such that each zone contains a measuring location. If we assume that the average water flux in each zone can be represented by the measurement at the respective measuring location, the overall water delivery rate in the sector can be calculated by

$$
\dot{V}=\sum_{k=1}^{K} \underset{k=1}{\Sigma} A_{p} \dot{v}_{k p}^{\prime \prime}
$$

where $\mathrm{P}$ is the number of measurements along a radius; $\mathrm{K}$ is the number of azimuthal measurements; $A$ is the area of the $p$-th zone in a radial direction; and $\dot{v}_{\mathrm{v}}$ is the flux at the location of the $\mathrm{p}-\mathrm{th}$ radial zone and the $\mathrm{k}-\mathrm{th}$ azimuthal angle. The delimitation of the divided zones along a radius was set at the center between the adjacent measuring positions. The same rule is employed to determine the delimiation along an arc. The edge of the outermost zone for each radius was delimited by the outer edge of the water collector.

Table II presents a comparison of the measured overall water delivery rates in the sector and the water delivery rates in the same area with axisymmetrical sprinkler sprays. In spite of the seemingly drastic variation of water flux from the bisector to the two sides of the sector, the water delivery rate based on axisymetry is close to (better than $84 \%$ ) the rate obtained by $\mathrm{Eq}$ (1).

TABLE II. Overa11 Water Delivery Rates, Median Drop Sizes, and C Constants for Eq. (4) in the 100-Degree Sector

\begin{tabular}{|c|c|c|c|c|}
\hline Case & $\begin{array}{l}\text { Actual Rates } \\
\text { in the Sector } \\
\text { (liters/min) }\end{array}$ & $\begin{array}{l}\text { Rates in a } 100^{\circ} \text { Sector } \\
\text { for Axisymmetrical Sprays } \\
\text { (1iters/min) }\end{array}$ & $\mathrm{d}_{\mathrm{m}}(\mathrm{mm})$ & $\underline{C}$ \\
\hline 1 & 66.4 & 63.1 & 1.66 & 4.30 \\
\hline 2 & 90.8 & 87.3 & 1.37 & 4.41 \\
\hline 3 & 68.7 & 63.1 & 1.61 & 4.17 \\
\hline 4 & 97.5 & 87.3 & 1.39 & 4.48 \\
\hline 5 & 53.6 & 45.2 & 0.96 & 2.86 \\
\hline 6 & 51.1 & 45.2 & 1.00 & 2.97 \\
\hline 7 & 38.2 & 32.6 & 0.86 & 2.33 \\
\hline
\end{tabular}

\subsection{Local and Gross Drop Size Distribution}

Figures 4 and 5 illustrate the typical radial and azimuthal variations of drop size distribution measured in the present study, respectively, using 


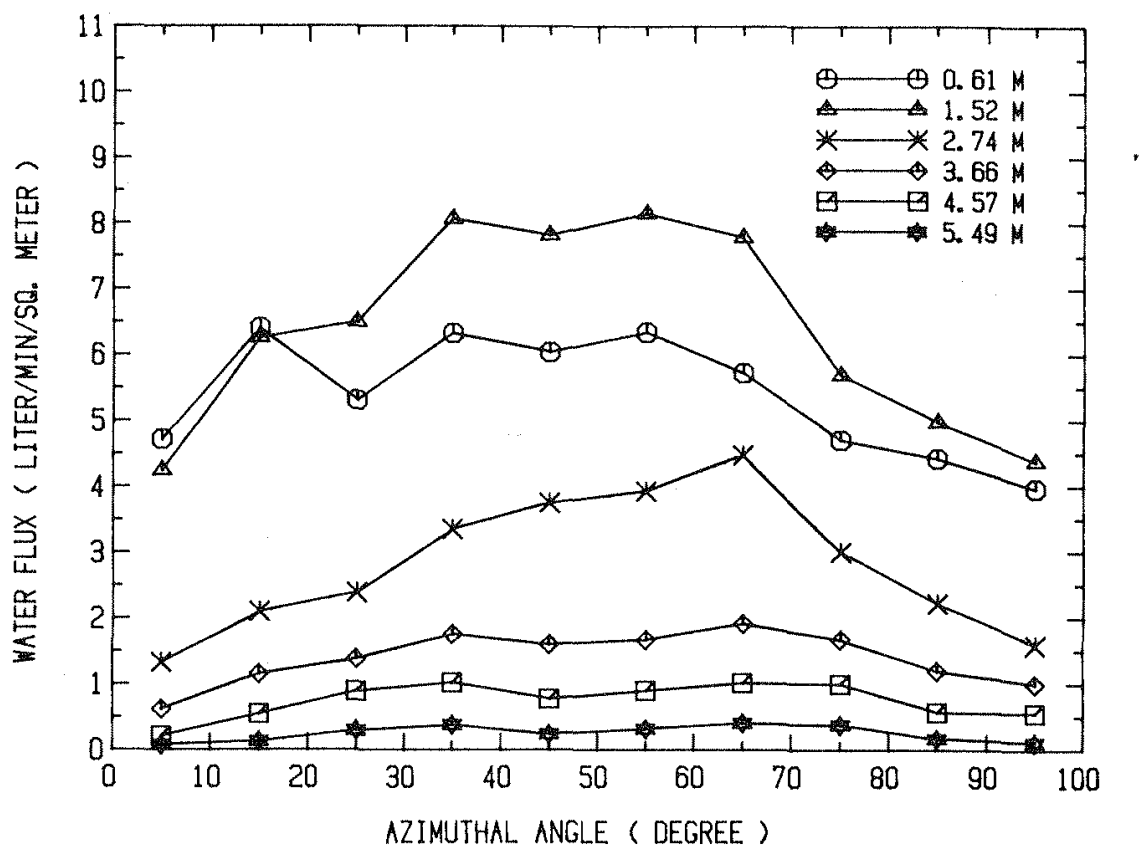

FIGURE 3. Spatial water flux distribution for Case 5.

Case 5 for examples. The measurements of drop size distribution of all seven tested cases are documented in Reference 5. The ordinate of Figures 4 and 5 represents the accumulative percents by volume (APV) below a drop size. Figure 4 shows that increasingly larger drops occur in the distribution with greater radial distance from the sprinkler. Figure 5 shows that small droplets tend to concentrate in the middle of the sector; correspondingly, increasingly larger drops exist toward the two sides of the sector. The actual factors causing drop size and water flux to systematically vary from the central region to the two sides of the sector are not fully understood. However, it is suspected that the effect of the pipe and the sprinkler's two arms on the initial spray formation might be responsible for producing the spray pattern found in this study.

Gross drop size distribution within the 100-degree measuring sector can be derived from the local water flux and drop size distributions measured in this area. The APV below a specified drop size can be written as:

$$
\text { APV }_{\ell}=\frac{\sum_{k=1}^{K} \sum_{p=1}^{P} A_{p} \dot{V}_{k p}^{\prime \prime} A_{k p \ell}}{\dot{V}}
$$




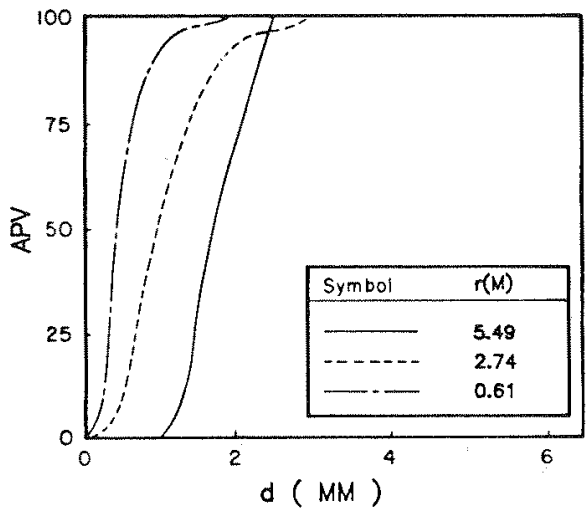

FIGURE 4. Radial variation of drop size distribution along the 45-degree radius for Case 5 .

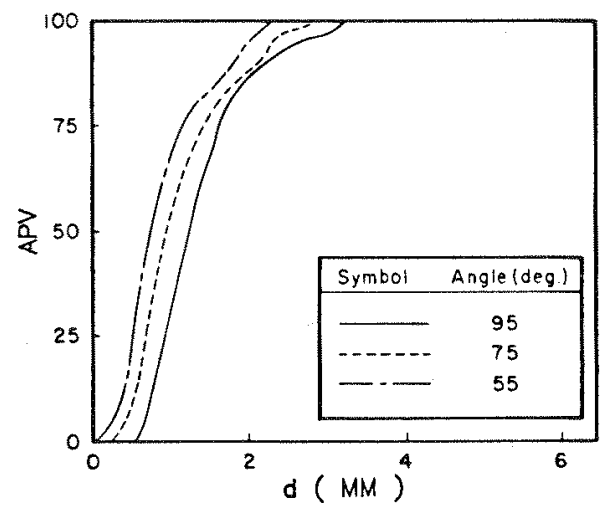

FIGURE 5. Azimuthal variation of drop size distribution at a radial distance of $2.74 \mathrm{~m}$ for Case 5 .

where $\mathrm{APV}_{\ell}$ is the gross APV below a drop size $\mathrm{d}_{\ell}$; and $\mathrm{APV}_{\mathrm{kp} \ell}$ is the $10 \mathrm{cal} \mathrm{APV}$ at the $k$-th azimuthal angle and the $p$-th radial zone.

The present results indicate that, with the same sprinkler type and water pressure, the gross drop size distribution 3.05 below the sprinkler can be reasonably reproduced at a vertical distance of $6.10 \mathrm{~m}$ below the sprinkler. Figure 6 demonstrates this finding by the gross drop size distributions of Gases 2 and 4, i.e., with a nozzle size of $16.3 \mathrm{~mm}$ and water pressure of $393 \mathrm{kPa}$. The gross size distributions of all seven tested cases are tabulated in Reference 5. The good agreement of gross drop size distributions obtained at the above two elevations implies that under the present test conditions, the process of drop formation is completed at $3.05 \mathrm{~m}$ below the sprinklers, and the net effects of drop breakup and coalescence, if any, is negligible below $3.05 \mathrm{~m}$.

For geometrically similar nozzles, Heskestad ${ }^{7}$ found that at room temperature the characteristic drop size can be related to the orifice size $D$ and water pressure $\Delta \mathrm{P}$ by the following expression:

$$
\mathrm{d} \sim \Delta \mathrm{P}^{-1 / 3} \mathrm{D}^{2 / 3}
$$

Equation (3) can be written in the following nondimensional expression by introducing the Weber number, $W e=\rho_{W} \mathrm{U}^{2} \mathrm{D} / \sigma_{\mathrm{W}}$ :

$$
\frac{\mathrm{d}_{\mathrm{m}}}{\mathrm{D}}=\mathrm{C} / \mathrm{We}^{1 / 3}
$$


where $d_{m}$ is the median drop size, $U$ is the water discharge velocity, and $\rho_{W}$ and $\sigma_{w}$ are water density and surface tension, respectively.

Table If tabulates the volumetric median drop sizes and associated $\mathrm{C}$ constants for all tested cases. The table indicates that, for a specific sprinkler, 1.e., Cases 1, 2, 3, and 4, the C values are about the same for different water pressures. Based on the present limited data, the pressure effect on drop size indicated in Eq (3) appears to be valid for sprays of commercial sprinklers.

\subsection{Correlations of Gross Drop Size Distribution}

It was found that the gross drop size distribution obtained from the present study could not be fitted entirely by either log-normal or Rosin-Rammler distribution ${ }^{8}$ as postulated in many previous applications of sprinkler sprays. To substantiate this finding, it was decided to fit the present data with both $\log$-normal and Rosin-Rammler distributions in their applicable size ranges.

The equation of $\log$-normal distribution is

$$
y=\frac{1}{\mathrm{~d} \sigma(2 \pi)^{1 / 2}} \exp \frac{-\left[\ln \left(\mathrm{d} / \mathrm{d}_{\mathrm{m}}\right)\right]^{2}}{2 \sigma^{2}}
$$

where $y$ is the occurring probability at drop size $d$, and $\sigma$ is the variance of the distribution. The units of $d, d_{m}$, and $\sigma$ are in millimeters. The APV below a drop size could not be obtained analytically from Eq (5) but by numerical integration. The accumulative percents of a log-normal distribution should be in a straight line if plotted on log-probability graph paper.

The Rosin-Rammler equation is

$$
y=\gamma k d^{\gamma-1} \operatorname{Exp}\left[-k^{\gamma}\right]
$$

where $k$ is a constant, and $\gamma$ is the constant which depends on the characteristics of a spray nozzle.

Integrating $\mathrm{Eq}$ (5), we obtain:

$$
\operatorname{APV}_{\ell}=1-\operatorname{Exp}\left[-\phi\left(\mathrm{d}_{\ell} / \mathrm{d}_{\mathrm{m}}\right)^{Y}\right]
$$

where $\phi=\mathrm{kd}_{\mathrm{m}}^{\gamma}$.

The data correlations for Case 2 are shown in Figure 6 . The data in the linear portion were fitted with Eq (5), while those in the curved position were correlated with Eq (7). Both the fitted straight line and curve are extended to the whole range of the measured drop sizes, to substantiate the deviations of the above two distribution patterns from the portions of data where they cannot be applied. 


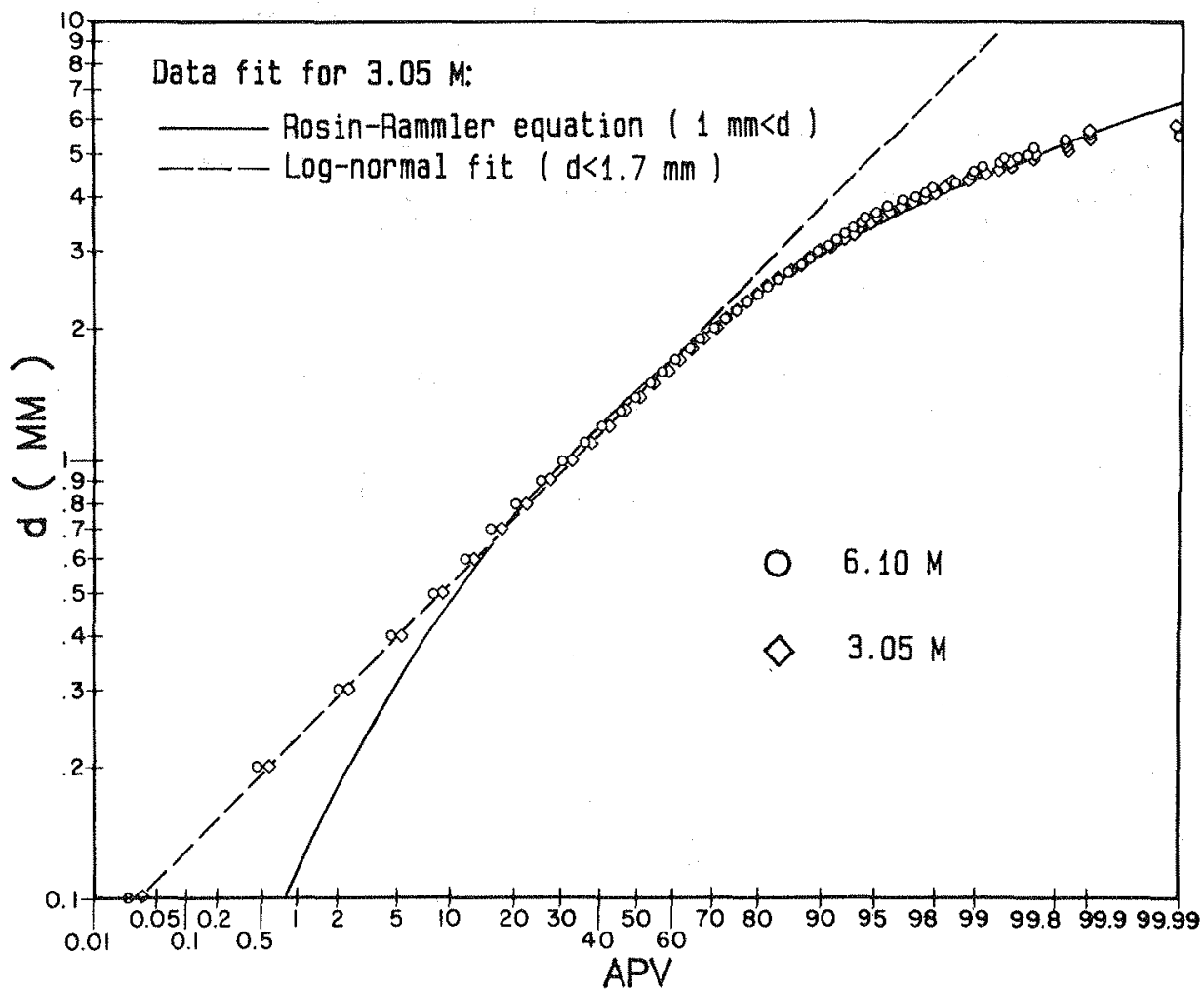

F IGURE 6. Gross drop size distributions for Cases 2 and 4 and data fit for Case 2.

Table III presents the parameters of $\sigma, \phi$, and $\gamma$ of Eqs (5) and (7), and their respective applicable drop size ranges.

TABLE III. The Values of $\sigma, \phi, \gamma$

$\begin{array}{cccccr}\text { Case } & \sigma(\mathrm{mm}) & \text { Range }(\mathrm{mm}) & \phi & \gamma & \text { Range (mm) } \\ 1 & 0.76 & 0.1-2.0 & 0.66 & 1.78 & 1.0-5.8 \\ 2 & 0.78 & 0.1-1.7 & 0.67 & 1.67 & 1.0-5.7 \\ 3 & 0.73 & 0.1-2.0 & 0.61 & 1.69 & 1.0-5.9 \\ 4 & 0.75 & 0.1-1.7 & 0.64 & 1.68 & 1.0-5.4 \\ 5 & 0.58 & 0.1-1.7 & 0.70 & 1.69 & 1.0-3.9 \\ 6 & 0.56 & 0.1-1.4 & 0.66 & 1.73 & 1.0-4.1 \\ 7 & 0.62 & 0.1-1.5 & 0.68 & 1.54 & 1.0-3.9\end{array}$




\subsection{A Proposed Tentative Test Procedure to Estimate Gross Drop Size Distri-} bution with Few Measurements

From the economic standpoint, it is desirable that the gross drop size distribution in an area of interest be closely estimated by conducting only a few measurements at strategic locations instead of mapping out the entire region.

On our present domain of measurements, the drop size was found to increase progressively with radial distance; drops in the bisector region were generally smaller than those at the two sides of the measuring sector. If we employ only the data along the bisector radius, the resulting distribution will be biased toward the smaller size regime. On the other hand, if we employ measurements along either one of the two sides of the sector, the distribution will then be weighted toward the larger size regime. However, a method may be developed by properly weighting the extreme spray properties at selected locations (i.e., along the bisector radius and either side of the present measuring sector) with their respective water flux. The water flux and local drop size distribution were generally not exactly symmetrical with respect to the bisector ${ }^{6}$; therefore, the present proposed scheme is to weight the spray properties of the two sides with their water fluxes at corresponding radial locations; i.e.:

$$
\begin{aligned}
& \dot{\mathrm{v}}_{\mathrm{e}, \mathrm{p}}^{\prime \prime}=\dot{\mathrm{v}}_{5, \mathrm{p}}^{\prime \prime} \frac{\dot{\mathrm{v}}_{5, \mathrm{p}}^{\prime \prime}}{\dot{\mathrm{v}}_{5, \mathrm{p}}^{\prime \prime}+\dot{\mathrm{v}}_{95, \mathrm{p}}^{\prime \prime}}+\dot{\mathrm{v}}_{95, \mathrm{p}} \frac{\dot{\mathrm{v}}_{95, \mathrm{p}}^{\prime \prime}}{\dot{\mathrm{v}}_{5, \mathrm{p}}+\dot{\mathrm{v}}_{95, \mathrm{p}}^{\prime \prime}} \\
& \mathrm{APV}_{\mathrm{e}, \mathrm{p} \ell}=\left(\mathrm{APv_{5,p \ell }} \dot{\mathrm{v}}_{5, \mathrm{p}}^{\prime \prime}+\mathrm{APV}_{95, \mathrm{p} \ell} \dot{\mathrm{v}}_{95, \mathrm{p}}^{\prime \prime}\right) /\left(\dot{\mathrm{v}}_{5, \mathrm{p}}^{\prime \prime}+\dot{\mathrm{v}}_{95, \mathrm{p}}^{\prime \prime}\right)
\end{aligned}
$$

where 5 and 95 represent the 5-degree and 95-degree radii respectively. The gross drop size distribution can now be calculated with the data from the 50-degree radius (the bisector) and the combined properties of the two sides by:

$\mathrm{APV}_{\ell}=\sum_{\mathrm{p}=1}^{\mathrm{P}} \mathrm{A}_{\mathrm{p}}\left(\mathrm{APV}_{50, \mathrm{p} \ell} \dot{\mathrm{v}}_{50, \mathrm{p}}^{\prime \prime}+\mathrm{APV}_{\mathrm{e}, \mathrm{p} \ell} \dot{\mathrm{v}}_{\mathrm{e}, \mathrm{p}}^{\prime \prime}\right) \mathrm{\Sigma}_{\mathrm{p}=1}^{\mathrm{p}} \mathrm{A}_{\mathrm{p}}\left(\dot{\mathrm{v}} \ddot{50, p}+\dot{\mathrm{v}}_{\mathrm{e}, \mathrm{p}}^{\prime \prime}\right)$

Figure 7 illustrates the gross drop size distributions for Case 7 derived from detailed measurements within the sector, as well as the distribution from the 50-degree radius only, and the combined result from the 5-, 50-, and 95-degree radii. Comparisons for the other tested cases are shown in Reference 5. In general, the combined results closely represent those obtained from detailed measurements and apparently are better than the estimation using data only from the 50-degree radius (the bisector).

\section{CONCLUSIONS}

Characteristics of single sprinkler sprays for three selected upright sprinklers were investigated with FMRC drop size measuring system. Sprinklers with nominal orifice sizes of $16.3,13.5$, and $12.7 \mathrm{~mm}$ were tested at water pressures of 206 and $393 \mathrm{kPa}$. Distributions of water flux and drop size distribution were mapped out in sectors at vertical distances of 3.05 and 
$6.10 \mathrm{~m}$ below the sprinkler. In the present study, the bisector of the sector was perpendicular to the imaginary plane containing the two sprinkler arms which were in alignment with the supporting pipe. A tentative test protocol was developed to obtain gross drop size distribution without mapping out the entire spray region. Several observations and findings are drawn from the present investigation:

1) Under the measuring conditions of this study, the water flux is roughly symmetrical to the bisector of the measurement domain. The water flux tends to decrease from the bisector to the two sides of the sector.

2) Large drops gradually increase their proportional contribution to the water flux as the radial distance is farther from the sprinkler. Azimuthally, the same trend is observed moving from the bisector toward the two sides of the sector.

3) The gross drop size distribution at $3.05 \mathrm{~m}$ below the tested sprinklers can be reproduced by measurements at the $6.10 \mathrm{~m}$ level.

4) Based on present results, the gross drop size distributions of the tested upright sprinklers correspond to a composite of two different distributions: the log-normal distribution for small drops and the Rosin-Rammler distribution for large drops (above the median size).

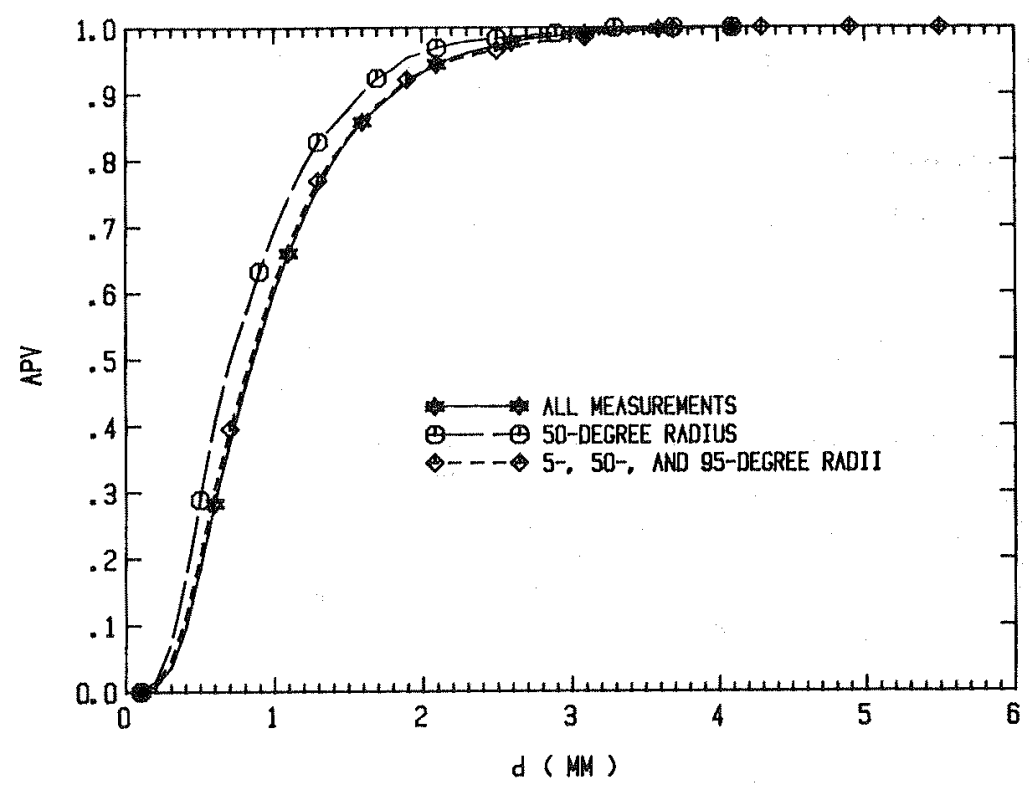

FIGURE 7. Comparison of three derived gross drop size distributions for Case 7 calculated from three different combinations of data. 


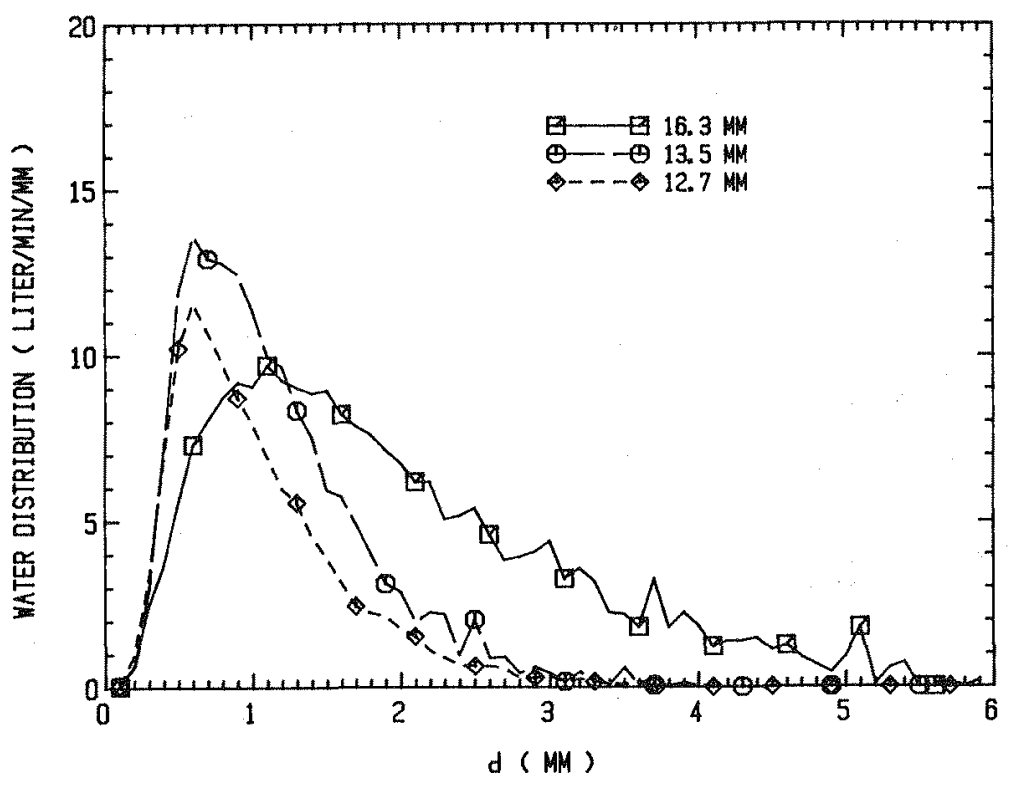

FIGURE 8. Water distribution as a function of drop size at $3.05 \mathrm{~m}$ below the sprinklers, with water pressure of $206 \mathrm{kPa}$.

5) Under the same test conditions, sprinklers with larger orifice produce larger drops than sprinklers with smaller orifice, regardless of difference in sprinkler design. Therefore, if greater penetrability of drops into the fire plume is desired, sprinklers with large orifice should be considered. On the other hand, if cooling ability is emphasized (small drops), sprinklers with small orifice should be used. Figure 8 demonstrates the trend of drop size distribution for the present three tested sprinklers at a water pressure of $206 \mathrm{kPa}$ and at $3.05 \mathrm{~m}$ below the sprinklers.

6) Based on the present limited data, the median drop size of a sprin$k$ ler appears to vary inversely proportional to the one-third power of water pressure.

\section{AC KNOWLEDGMENTS}

The author gratefully acknowledges the support and encouragement of $\mathrm{Mr}$. C. Yao and Dr. H. C. Kung during the course of this work. Special thanks are extended to: Mr. A. P. Symonds for providing the maintenance service for the FMRC Drop Size measuring system; Mr. W. R. Brown for designing and fabricating the probe traversing apparatus; Mr. E. E. Hill for assistance in constructing the probe traversing apparatus; and $\mathrm{Mr}$. D. E. Charlebois and the Factory Mutual Test Center staff for assembling the test apparatus and for assistance in conducting the tests. 
1. Knollenberg, R. G.: "The Optical Array: An Alternative to Scattering on Extinction for Airborne Particle Size Determination," J. Applied Meteorology, 9, $86-103,1970$.

2. Croce, P.A., You, H.Z., Khan, M.M., and Symonds, A.P.: "Calibration of, and Preliminary Measurements with, FMRC's PMS Drop-Size Measuring System," FMRC Technical Report, J.I. OF OE2.RA, 1981.

3. You, H.Z. and Symonds, A.P.: "Sprinkler Drop-Size Measurements, Part I: An Investigation of the FMRC PMS Drop-Size Measuring System," FMRC Technical Report, J.I. OG1E7.RA, 1982.

4. Khan, M.M.: "Particle Measuring Systems: Operation Manual, "FMRC Internal Memo, 1981.

5. You, H.Z.: "Sprinkler Drop-Size Measurements, Part II: An Investigation of the Spray Patterns of Selected Commercial Sprinklers with the FMRC PMS Droplet Measuring System," FMRC Technica1 Report, J.I. OG1E7.RA 1983.

6. Goodfellow, D.: "Apparatus and Procedure for Measuring the Actual Delivered Density of Sprinklers in Simulated Rack Storage Fires, Volume I - Apparatus and Procedure Development," FMRC Technical Report, J.I. OK0J4.RR, 1984.

7. Heskestad, G.: "Proposal for Studying Interaction of Water Sprays with Plume in Sprinkler Optimization Program," FMRC Interoffice Correspondence, 1972.

8. Rosin, P. and Rammler, E.: "The Laws Governing the Fineness of Powdered Coa1," The Institute of Fue1, 29-36, October, 1933. 